\title{
The Viscosity of Liquids.
}

\begin{abstract}
AMONG the properties of liquids the viscosity is probably the one the investigation of which has suffered most from lack of any accepted theory, however crude and approximate, to guide it. A great body of more or less careful observations exist, but it has furnished remarkably little information as to the nature of the liquid state. The new technique of X-rays, the Raman effect, and the depolarisation of light may do much to elucidate the structure of liquids, but the older and grosser property of viscosity must be at least as pertinent. Recently, a letter of mine published in NaTuRE of Mar. 1 upon the subject of liquid viscosity called forth a number of letters, and seemed to make it advisable to say a little more of a theory which, little elaborated as it is, offers a picture which may prove helpful. The conception of a transitory and fluctuating 'crystallisation' of a liquid seems to fit in with other observations. I am far from being satisfied with the theory as it stands: my hopes go no further than that the facts cited in my present letter may suggest to some that there is a germ of truth in the point of view put forward. At any rate, I intend to make some measurements myself of the temperature coefficient of liquid viscosity, in the hope that they may throw some light on the old problem as to the force exerted on a single molecule by the molecules in its irnmediate neighbourhood, within the Lorentz sphere.
\end{abstract}

The letter of Prof. Andrade in Nature of Mar. 1 raises the interesting question as to the reason for the decrease in viscosity of a liquid with rise of temperature. This seems to be closely connected with the similar decrease in the frictional resistance due to the motion of solid bodies through liquids. I have been lately carrying out some experiments on this latter subject as follows.

Two rings of thin sheet-brass were cut off a drawn brass telescope tube about 2 inches in diameter. One ring was 1 inch deep and the other 0.25 inch deep. These rings could be suspended one at a time by four very fine wires with their axes vertical from the bottom of a cylindric inertia mass, which last was hung by a single steel torsion wire. The arrangement was such that the thin rings could be set oscillating in a liquid successively, with the rings totally immersed in it, but the inertia mass not immersed. The object of using rings of different depths was to eliminate any energy loss due to wave or eddy current making due to the fine suspending wires or edges of the rings. The measurement consisted in displacing the suspended mass through a certain angle, say $120^{\circ}$, and then noting the time required for this angle of maximum displacement to be reduced to $60^{\circ}$ for the two rings. This gives by difference a measure of the frictional resistance per unit of surface. If the frictional resistance were exactly proportional to the velocity then the differential equation for the motion would be

$$
I \frac{d^{2} \theta}{d t^{2}}+R \frac{d \theta}{d t}+C \theta=0
$$

where $I$ is the moment of inertia of the oscillating mass and $C$ the coefficient of restoration and $\theta$ the angle of displacement. If $t^{\prime}$ is the time required to decrease the initial amplitude of displacement to half its value, then it is easy to show that $R=1 \cdot 38 I / t^{\prime}$.
On making the measurements in tap water at various temperatures the following results were found :

$\begin{array}{lclr}15^{\circ} \mathrm{C} . & R=19 \cdot 75 & 60^{\circ} \mathrm{C} . & R^{\prime}=10 \cdot 1 \\ 30^{\circ} & 15 \cdot 2 & 70^{\circ} & 10 \cdot 7 \\ 50^{\circ} & 12 \cdot 1 & 80^{\circ} & 10.6\end{array}$

It is seen that a rise of temperature of the water from $15^{\circ} \mathrm{C}$. to $80^{\circ} \mathrm{C}$. reduces the frictional coefficient $R$ to about half its value.

This method is sufficiently sensitive to show the difference between fresh water and sea water at the same temperature.

If we ask the reasons for it the following suggest themselves. The cause of friction between a solid and a liquid may be regarded as of the same nature as the reason for the coherence of molecules together to form either a solid or a liquid. In view of the assumed electric structure of atoms, this coherence must be regarded as due to electrical attractions between atoms or molecules. Rise of temperature ionises or dissociates molecules into ions or atoms and promotes mobility of these with respect to the mass of the liquid. Hence follows a reduction of the force required to shear a liquid surface along a solid.

Experiments made with paraffin oil (Royal Day. light) showed that, although this liquid seems more limpid than water, the coefficient of frictional resistance with the brass rings was considerably greater. The oil is, however, a good dielectric and therefore not ionised so much as tap water at the same tem. perature. The practical result is that if the sea had an average temperature of $80^{\circ} \mathrm{C}$. instead of about $15^{\circ} \mathrm{C}$., ships would require less power to drive them through the water than at present. I am continuing these experiments as time permits.

Ambrose Fleming.

Manor Road, Sidmouth, Mar. 1.

I HAve read with great interest Prof. Andrade's letter in NATURE of Mar. 1, dealing with the temperature variation of the viscosity of liquids. The theoretical side of this question has admittedly been curiously neglected with perhaps one or two exceptions, of which one of the earliest was Maxwell. Considering, however, how generally the idea of a time of relaxation has entered into modern molecular theory, particularly in relation to liquids, it is more than probable that Maxwell's original conception of the viscosity process will receive an elaborated physical interpretation.

In view of this fact I should like to mention a formula connecting the viscosity and temperature in liquids which I have had occasion to examine in a paper which has been communicated elsewhere, and which may be built up from Maxwell's fundamental definition of liquid viscosity. This formula

$$
\eta=\frac{A e^{\beta T}}{\bar{T}-b}
$$

holds for a number of liquids over a wide temperature range, which liquids include both normal and associating liquids. The accompanying table shows two examples. The formula has admittedly three constants but is similar to that of Prof. Andrade in that it contains an exponential function. From general molecular theory and in view of the direct relation between viscosity and vapour pressure, it is probable that an exponential form of equation

No. 3154 , VoL. 125] 
for viscosity and temperature will prove to be the most satisfactory. In this connexion a comparison of the derivations of J.S. Dunn's equation (Trans.

\begin{tabular}{|c|c|c|c|c|}
\hline \multirow[t]{2}{*}{ Temp. } & \multicolumn{2}{|c|}{ Water, $\begin{array}{l}A=1.522 . \\
\beta=-0.003822 . \\
b=243\end{array}$} & \multicolumn{2}{|c|}{ Octane, $\begin{array}{l}A=1.128 \\
\beta=-0.002399 \\
b=190 .\end{array}$} \\
\hline & $\eta$ obs. & $\eta$ calc. & $\eta$ obs. & $\eta$ cale. \\
\hline $0^{\circ} \mathrm{C}$. & 0.01792 & 0.01787 & 0.007060 & 0.007060 \\
\hline 10 & 01308 & 01289 & 006159 & 006152 \\
\hline 20 & 01005 & 00996 & 005419 & 005423 \\
\hline 30 & 00801 & 00797 & 004828 & 004826 \\
\hline 40 & 00656 & 00657 & 004328 & 004328 \\
\hline 50 & 00549 & 00554 & 003907 & 003908 \\
\hline 60 & 00469 & 00474 & 003551 & 003548 \\
\hline 70 & 00406 & 00410 & 003241 & 003238 \\
\hline 80 & 00357 & 00359 & 002971 & 002967 \\
\hline 90 & 00317 & 00317 & 002730 & 002730 \\
\hline 100 & 00284 & 00281 & 002520 & 002519 \\
\hline 110 & - & - & 002335 & 002332 \\
\hline 120 & _. & 一 & 002160 & 002165 \\
\hline
\end{tabular}

The values for $\eta$ obs. for water are those of Bingham and Jackson, for octane those of Thorpe and Rodger.

Farad. Soc., 22, pp. 401-405; 1926) $1 / \eta=A e^{-Q / R T}$ based on kinetic theory considerations and $\eta=A e^{b / T}$ put forward by Prof. Andrade should be of great interest.

\section{Crowther Road, Stockland Green, Erdington, Birmingham.}

\section{E. W. MADGE.}

A SATISFACTORY explanation of the decrease of liquid viscosity with temperature has long been required, and Prof. Andrade's theoretical treatment of the subject will be awaited with interest. His simple formula connecting viscosity and temperature appears to give good agreement for certain liquids, but I should like to point out that it does not give such good agreement when applied to measurements on some mineral oils, as does a formula due to Slotte. The measurements were taken several years ago and in attempting to find an empirical relationship between the viscosity and the temperature, I obtained, quite independently, a formula which was identical

\begin{tabular}{|c|c|c|c|}
\hline Temp. & $\eta$ calc. (Andr.) & $\eta$ obs. & $\eta$ calc. (Slotte) \\
\hline 20 & $12 \cdot 36$ & $15 \cdot 20$ & $15 \cdot 46$ \\
25 & $9 \cdot 03$ & $10 \cdot 15$ & $10 \cdot 19$ \\
30 & $6 \cdot 67$ & $7 \cdot 04$ & $6 \cdot 99$ \\
35 & $4 \cdot 97$ & $4 \cdot 97$ & $4 \cdot 93$ \\
40 & $3 \cdot 75$ & $3 \cdot 58$ & $3 \cdot 58$ \\
45 & $2 \cdot 85$ & $2 \cdot 67$ & $2 \cdot 66$ \\
50 & $2 \cdot 18$ & $2 \cdot 00$ & $2 \cdot 01$ \\
55 & $1 \cdot 685$ & $1 \cdot 550$ & $1 \cdot 558$ \\
60 & $1 \cdot 313$ & $1 \cdot 232$ & $1 \cdot 217$ \\
65 & $1 \cdot 028$ & $0 \cdot 965$ & $0 \cdot 966$ \\
70 & $0 \cdot 813$ & $0 \cdot 780$ & $0 \cdot 777$ \\
75 & $0 \cdot 646$ & $0 \cdot 633$ & $0 \cdot 636$ \\
80 & $0 \cdot 517$ & $0 \cdot 517$ & $0 \cdot 522$ \\
85 & $0 \cdot 417$ & $0 \cdot 432$ & $0 \cdot 435$ \\
90 & $0 \cdot 337$ & $0 \cdot 362$ & 0.363 \\
95 & $0 \cdot 275$ & $0 \cdot 306$ & $0 \cdot 305$ \\
\hline
\end{tabular}

with that due to Slotte and adopted by Thorpe and Rodger as best representing their experimental results. This formula is usually written in the form

$$
\eta=\frac{c}{i+a} n
$$

where $\eta$ is the viscosity, $t$ the temperature in ${ }^{\circ} \mathrm{C}$., and $C, a$ and $n$ constants depending on the liquid used. The constants $A$ and $b$ in Prof. Andrade's formula
(NATURE, Mar. 1, p. 309) have been calculated for a somewhat viscous oil from the viscosity values at $35^{\circ} \mathrm{C}$, and $80^{\circ} \mathrm{C}$, and the agreement between the observed results and those calculated from the two formulæ shown in the accompanying table.

One naturally expects to be able to obtain closer agreement between observed and calculated results with a formula containing three constants than with one containing two, but in this connexion a further interesting fact was observed. It was found that the value of $C$ obtained from eight oils of widely vary. ing viscosities could be expressed with considerable accuracy by the formula

$$
C=a d^{n}
$$

where $a$ and $d$ are constants which are independent of the nature of the oil and the approximate values of which were $1.5 \times 10^{-3}$ and 400 respectively. Thus Slotte's formula also becomes one in which there are only two constants the values of which are dependent on the nature of the liquid. On examining the values of $C$ and $n$ obtained for other liquids it was found that, with the exception of the alcohols, they were connected by a similar relationship, the values of $a$ and $d$ differing but slightly from those obtained for the mineral oils. This empirical relationship was not published, and it was thought that the results might be of some interest when considering the theoretical aspects of the problem.

\section{International Telephone and Telegraph} Laboratories, Incorporated, The Hyde, Hendon.

In a recent issue of Nature (Mar. 1, p. 309) Prof. Andrade publishes the following formula, $\eta=A e^{b / T}$, for the coefficient of viscosity of liquids as a function of the temperature $T$, which he shows to be in excellent agreement with the experimental data. Now I wish to point out that a practically equivalent formula, namely, $\eta=C T e U / k T$, was given by me more than four years ago in a paper on the heat motion of solid and liquid bodies (Zeit. f. Phys., 35, p. 664-667; 1926). Since this has obviously remained unnoticed, it may be well to state briefly the fundamental conception on the heat motion of liquids upon which the above formula is based.

The 'crawling' of the particles of the liquid is considered as a combination of oscillations about a (temporary) position of equilibrium and of a jerky displacement of this position from time to time. The average number of oscillations performed about the same equilibrium position is equal to $e U / k T$, where $u$ is the energy required to tear the particle out of it. An elementary displacement of the equilibrium position is consequently achieved in a time $\tau=\tau_{0} e U / k T, \tau_{0}$ being the period of the oscillations, which corresponds to a ' crawling' velocity $v=\frac{\delta}{\tau}=\frac{\delta}{\tau_{0}} e-U / k T$, where $\delta$, the average range of this displacement, is of the order of the mean distance between neighbouring particles, and to a diffusion coefficient $D=\frac{1}{3} \delta v=\frac{\delta^{2}}{3 \tau_{0}} e-v / k T$. Now the latter is connected with the friction coefficient $f(=$ ratio of force to the mean velocity which is due to it) by Einstein's formula $D f=k T$. If, on the other hand, we regard the particle as a small sphere of radius $a \cong \delta$ then we have by Stokes's formula $f=6 \pi a \eta$. Hence $\eta=\frac{\tau_{0} k T}{2 \pi a \delta^{2}} e^{U / k T}$ which is the above formula with $C=\frac{\tau_{0} k}{2 \pi a \delta^{2}}$.

No. 3154 , VoL. 125] 